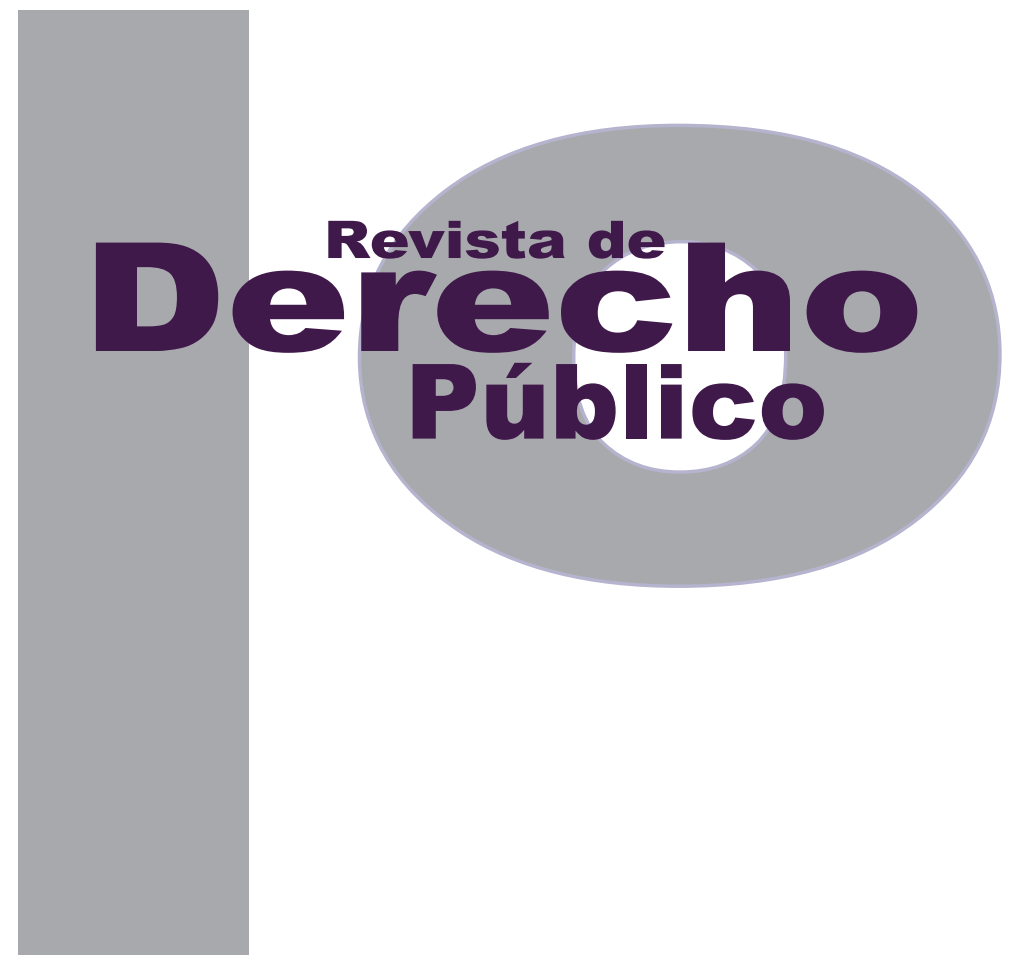

\title{
EL USO DE AERONAVES REMOTAMENTE TRIPULADAS EN EL CONFLICTO ARMADO COLOMBIANO Y EL DERECHO INTERNACIONAL HUMANITARIO
}

\author{
Manuel Guillermo Contreras Henao
}

DOI: http://dx.doi.org/10.15425/redepub.33.2014.10

Universidad de los Andes

Facultad de Derecho

Revista de Derecho Público N. ${ }^{\circ} 33$

Julio - Diciembre de 2014. ISSN 1909-7778 


\title{
El uso de aeronaves remotamente tripuladas en el conflicto armado colombiano y el derecho internacional humanitario
}

\section{Resumen}

La evolución de la tecnología de las armas ha representado un reto para la aplicación del derecho internacional humanitario. En este marco, el uso de aeronaves remotamente tripuladas en conflictos armados ha resultado controversial y se ha cuestionado en diferentes escenarios. Recientemente, las Fuerzas Militares de Colombia adquirieron esta tecnología, con la posibilidad de armar estas aeronaves y usarlas en el conflicto armado. En este texto se estudian las implicaciones del uso de esta tecnología en el país, y se concluye que siempre y cuando su uso se haga de acuerdo con los preceptos del derecho internacional humanitario, no tiene por qué representar un riesgo para la trasgresión del régimen jurídico de los conflictos armados.

Palabras clave: derecho internacional humanitario, conflicto armado colombiano, conflicto armado interno, Convención de Ginebra, Protocolo Adicional II, aeronaves remotamente tripuladas, vehículos aéreos no tripulados, drones.

\section{The use of unmanned aerial vehicles in the Colombian armed conflict and International Humanitarian Law}

\begin{abstract}
The evolution of weapon technology has represented a great challenge for the implementation of International Humanitarian Law. As such, the use of unmanned aerial vehicles in armed conflict has been controversial, and has been criticized under different scenarios. Recently, the Colombian Military has acquired this technology, with the possibility of arming these aircraft and using the in the Colombian internal conflict. This text studies the implications of using this technology in Colombia, and concludes that as long as as they are used in accordance to the mandates of international humanitarian law, it does not constitute a violation of the law of armed conflicts.
\end{abstract}

Keywords: International Humanitarian Law, Colombian armed conflict, internal armed conflict, Geneva Convention, Additional Protocol II, unmanned aerial vehicles, drones.

\section{O uso de aeronaves remotamente tripuladas no conflito armado colombiano e o direito internacional humanitário}

\section{Resumo}

A evolução da tecnologia das armas tem representado um desafio para a aplicação do direito internacional humanitário. Neste marco, o uso de aeronaves remotamente tripuladas em conflitos armados tem resultado controverso e se tem questionado em diferentes cenários. Recentemente, as Forças Militares da Colômbia adquiriram esta tecnologia, com a possibilidade de armar estas aeronaves e usá-las no conflito armado. Neste texto são estudadas as implicações do uso desta tecnologia no país, e conclui-se que sempre e quando seu uso for de acordo com os preceitos do direito internacional humanitário, não tem por que representar um risco para a transgressão do regime jurídico dos conflitos armados.

Palavras-chave: direito internacional humanitário, conflito armado colombiano, conflito armado interno, Convenção de Genebra, Protocolo Adicional II, aeronaves remotamente tripuladas, veículos aéreos não tripulados, drones. 


\title{
El uso de aeronaves remotamente tripuladas en el conflicto armado colombiano y el derecho internacional humanitario*
}

\author{
Manuel Guillermo Contreras Henao**
}

\begin{abstract}
SUMARIO
Introducción - I. ¿QUÉ ES UNA AERONAVE REMOTAMENTE TRIPULADA? - II. AERONAVES REMOTAMENTE TRIPULADAS EN COLOMBIA Y LATINOAMÉRICA: ¿POR QUÉ Y PARA QUÉ? - A. Las ART en Latinoamérica - B. Las ART en Colombia - C. Riesgos del uso de ART en Colombia - III. EL DERECHO INTERNACIONAL HUMANITARIO Y LAS ART - A. Legalidad del uso de armas en los conflictos armados - B. Principio de distinción - C. Principio de proporcionalidad - IV. CUÁLES SON LOS RIESGOS DE OPERAR AERONAVES REMOTAMENTE TRIPULADAS? - V. ¿EL USO DE ART ARMADAS EN EL CONFLICTO ARMADO COLOMBIANO ES LEGAL Y CONSISTENTE CON EL DERECHO INTERNACIONAL HUMANITARIO? - VI. CONCLUSIONES - Referencias.
\end{abstract}

Cómo citar este artículo: Contreras Henao, M. G. (Diciembre, 2014). El uso de aeronaves remotamente tripuladas en el conflicto armado colombiano y el derecho internacional humanitario. Revista de Derecho Público, 33. Universidad de los Andes (Colombia).

** Abogado de la Universidad de los Andes con opción en Ciencia Política. Correo: mg.contrerash@gmail.com 
Introducción

Las aeronaves sin pilotos no son un nuevo avance en la tecnología aeronáutica, su existencia se remonta casi a los inicios de la aviación. De hecho, el artículo 8 del Convenio de Chicago de 1944 hace referencia a dichos vehículos y su regulación. Como tal, la utilización de aeronaves tripuladas de manera remota no es una novedad en la aviación. En muchos casos son usadas para experimentos de seguridad, es decir, para comprobar la seguridad de aeronaves comerciales, o inclusive como objetivos para que pilotos de combate realicen su entrenamiento.

No obstante, en los últimos veinticinco años, las aeronaves remotamente tripuladas (ART O UAV por sus siglas en inglés) han adquirido un rol en los campos de combate. Inicialmente, fueron utilizadas para dirigir fuego de artillería o inclusive cortas misiones de reconocimiento. ${ }^{1} \mathrm{Sin}$ embargo, esta función se ha hecho más importante recientemente, al destinarlas a misiones de reconocimiento por periodos extensos y para realizar bombardeos y destrucción de objetivos selectivos.

Es necesario señalar que los vehículos remotamente tripulados no necesariamente son aeronaves. Actualmente también existen vehículos terrestres y de superficie, cuya tripulación está ubicada remotamente. En este texto se hará referencia únicamente a las aeronaves tripuladas de manera remota, también conocidas como drones.

1 Misión por medio de la cual se recolecta información e inteligencia, útil para el desarrollo de operaciones militares.
Como ya se advirtió, esto representa un reto para el derecho internacional humanitario, pues en principio no es claro si el uso de este tipo de aeronaves en el combate está permitido por las normas del régimen internacional del conflicto armado. El derecho internacional humanitario dispone una serie de normas sobre qué tipo de armamento está permitido utilizar en los conflictos armados tanto internacionales como internos. Igualmente, se debe examinar si el uso de cierto tipo de armamento respeta los principios básicos del derecho internacional humanitario; específicamente los principios de distinción y proporcionalidad, los cuales resultan violentados con más frecuencia. De esta forma, se puede apreciar que no existe claridad sobre si un Estado puede hacer uso de las ART en desarroIlo de operaciones de reconocimiento, de apoyo aéreo cercano, bombardeo y destrucción de blancos selectivos (Bowden, 2013).

Estas preguntas se acercan más a la realidad colombiana, si se tiene en cuenta que las Fuerzas Militares recientemente adquirieron diferentes tipos de ART con el propósito de luchar contra los grupos armados al margen de la ley (El Tiempo, mayo 2013), al igual que han empezado a desarrollar su propia ART (El Tiempo, octubre de 2013). Tanto la Fuerza Aérea como el Ejército han utilizado ART con funciones de reconocimiento. Sin embargo, en los últimos años la Fuerza Aérea adquirió drones de fabricación israelí, con la capacidad de portar armas y desarrollar misiones de apoyo aéreo cercano para operaciones terrestres (Webinfomil.com, 2012). Estas aeronaves ya estarían Ilegando a Colombia, según reportes de prensa (Webinfomil.com, 
2013), por lo cual el estudio de la legalidad de su uso se convierte en un tema prioritario, para evitar posibles abusos a la normatividad internacional.

Un análisis superficial de este tipo de armamento indica que no existe diferencia sustancial entre una aeronave remotamente tripulada y una aeronave de combate convencional (Carafano, 2013), excepto el que su tripulación no se encuentra dentro de la plataforma de armas. Con base en ello, en este texto se busca responder a la interrogante de si el uso de aeronaves remotamente tripuladas en los conflictos armados, específicamente en el conflicto armado colombiano, trasgrede o no las normas establecidas por el derecho internacional humanitario. En consecuencia, se ahondará y se estudiará cómo el uso de drones entra en tensión con los principios del derecho internacional humanitario. Se señala, inicialmente, que la hipótesis a esta pregunta de investigación es que el uso de ART en el conflicto armado colombiano es consistente con las normas del derecho internacional humanitario, por lo cual su utilización es perfectamente legal.

Dado que no existen mayores fuentes jurídicas que señalen expresamente una respuesta a esta pregunta, se requiere una metodología de análisis más profunda. Por esta razón, se analizarán las normas de derecho internacional humanitario existentes, al igual que los pronunciamientos de tribunales internacionales, para compararlos con las elucubraciones de diferentes autores reconocidos y poder elaborar una respuesta clara a la pregunta de investigación.
Para lograr el objetivo anteriormente descrito, primero se explicarán las características técnicas de las aeronaves remotamente tripuladas en general, de modo que el lector pueda comprender el contexto y el porqué de la tensión entre su uso y la aplicación del derecho internacional humanitario. Luego, se indagará por qué es relevante estudiar el uso de este tipo de aeronaves en Colombia, haciendo un rápido análisis de la situación en el país y en Latinoamérica. Terminada esta explicación, se analizarán los elementos de derecho internacional que regulan el uso de armas y los métodos para hacer la guerra; concretamente se mirará qué dicen las normas del conflicto armado, la jurisprudencia de las Cortes internacionales y la doctrina acerca de la legalidad del uso de armas, haciendo un paralelo con las características de las ART. Posteriormente se estudiarán los riesgos que presenta la utilización de drones, y se explorarán argumentos que apoyan su uso, al igual que aquellos que lo critican. Con esto, se podrá determinar si el uso de drones en el conflicto armado colombiano es legal. Finalmente, se concluirá brevemente sobre lo expuesto, y se comprobará la hipótesis plantada estableciendo si es acertada o no.

\section{I. ¿QUÉ ES UNA AERONAVE REMOTAMENTE TRIPULADA?}

Explicar literalmente lo que es una ART es muy sencillo si solo se atiende a su nombre. Evidentemente, un vehículo aéreo no tripulado es una aeronave cuya tripulación no se encuentra a bordo de esta. Es decir, el vehículo puede es- 
tar en un punto $X$, mientras que su operador se encuentra en un punto $Y$, sin que interese la distancia que pueda existir entre estos dos (Bowden, 2013). Si bien esta es una de las diferencias más importantes entre las ART y las aeronaves convencionales, no es la única. Por eso, es necesario explicar lo que es un drone.

Sin embargo, antes de continuar con la explicación sobre lo que es una ART, es necesario entender para qué pueden ser utilizadas por las fuerzas militares de un determinado país. Al desarrollar una serie de roles en sede de un conflicto armado, pueden cumplir funciones de vigilancia, recopilando inteligencia sobre cierto sector, lo que es de alto valor para los líderes militares; igualmente, pueden desarrollar funciones de observación avanzada para dirigir fuego de artiIlería sobre objetivos militares (Bowden, 2013). Por otra parte, las ART pueden cumplir un rol bélico, a través de misiones de apoyo cercano para el combate, ${ }^{2}$ operaciones de bombardeo y destrucción de blancos selectivos ${ }^{3}$ (Cole y Wright, 2010). Este último papel atribuido a los drones es quizás uno de los más controversiales, por lo cual a lo largo de este texto se le prestará mayor atención. El primer ataque con ART utilizando armamento en contra de un objetivo se produjo el 4 de noviembre de 2002 en Afganistán. En esa ocasión, un drone de la Fuerza Aérea de Estados Unidos disparó un misil Hellfire contra el ve-

2 Estas misiones tienen la intención de apoyar a las tropas en tierra y eliminar objetivos de alto valor como infraestructura enemiga, unidades enemigas y líderes militares enemigos.

3 Misiones de las fuerzas armadas encaminadas a la destrucción de objetivos específicos como campamentos enemigos y cualquier tipo de infraestructura utilizada por estos para desarrollar y financiar sus operaciones. hículo en que se movilizaba el líder de Al-Qaeda, Abu Ali al-Harithi (Bowden, 2013).

Continuando con la explicación, en primer lugar se señala que los drones son aeronaves que navegan por el espacio aéreo bajo los mismos principios que los aviones convencionales, bien sean de ala fija (aviones) o ala rotatoria (helicópteros). Según Bowden: “[a]un cuando los drones varían en tamaño desde los pequeños Raven, lanzados al aire por unidades de infantería para que puedan ver qué ocurre en la otra loma, hasta el Global Hawk, que es aproximadamente del tamaño de un Boeing 737, el aparato en sí es una aeronave (2013).

Como ya se mencionó, la totalidad de la tripulación de las ART se encuentra en una locación diferente a la de estas, es decir, no están ocupadas por ninguna persona con capacidad para operar el vehículo ni sus armamentos (Cole y Wright, 2010).

Como causa de lo anterior, las ART suelen ser significativamente más ligeras que una aeronave convencional. En este sentido, gozan de una mayor autonomía de vuelo, es decir, pueden permanecer en el aire por más tiempo que una aeronave tripulada (El Mercurio, 2013). Esta característica les otorga una capacidad de vigilancia permanente (persistent stare capability) (McNab y Matthews, 2011, p. 691) sin necesidad de reabastecerse de combustible. Adicionalmente, dado que la tripulación se encuentra en lugares diferentes a los de la aeronave, generalmente en tierra, es posible que los pilotos hagan turnos y de esa manera eviten la fatiga, por lo cual se 
pueden hacer misiones considerablemente más largas (Bowden, 2013). Sin embargo, el que los pilotos vuelen menos horas con el fin de evitar el cansancio puede llevar a una serie de errores que ponen en riesgo la misión y la aeronave, pero asegura que estos puedan discernir mejor sobre su objetivo y tomen decisiones más acertadas (Carafano, 2013).

A fin de que las ART puedan ser operadas remotamente, es necesario que estén equipadas con una serie de sensores y cámaras de alto poder y resolución que les permitan a sus operarios ver hacia dónde se dirige el drone y lo que está ocurriendo en tierra, incluso reconocer rostros, y recolectar la mayor cantidad de información sobre una gran porción de terreno observado. La mayoría de los sistemas de video con que están dotadas posibilitan grabar la totalidad del vuelo, de modo que las cintas pueden ser estudiadas por analistas y precisar los datos recolectados por las aeronaves (Bowden, 2013).

Como se mencionó anteriormente, las ART son capaces de desarrollar una variedad de misiones, entre las cuales se encuentran misiones de reconocimiento, comunicaciones y ataques aéreos. En este último rol, los drones utilizados en Afganistán, Pakistán e Israel usaron armamento aire-tierra, generalmente misiles antitanque o bombas dirigidas por láser (Castranova, 2013). Con este tipo de armamento pueden impactar los objetivos desde mayor distancia y con mayor precisión, lo cual no solo asegura el éxito de la misión, sino que reduce significativamente la posibilidad de incurrir en daños colaterales. No obstante, las ART pueden portar una cantidad innumerable de armamento y cámaras, de acuerdo con las capacidades bélicas de cada Estado (Freidersdorf, 2014). Todas estas características hacen que los ejércitos quieran equiparlas con las armas más precisas posibles, para utilizar plenamente sus capacidades (Vogel, 2011).

A lo anterior se suma el que las ART no requieren infraestructura compleja para poder operar, como por ejemplo cierto tipo de pistas. Algunas clases de drones pueden ser lanzados desde catapultas y recuperados por mallas, lo cual facilita su operación en zonas de geografía accidentada, inclusive por parte de tropas de infantería (Bowden, 2013). Naturalmente, entre más grande sea la ART, mayor infraestructura va a necesitar para poder decolar y aterrizar (Cole y Wright, 2010).

De esta forma, es claro que los drones son aeronaves especiales, con características que los diferencian de aviones tradicionales. Por ser sistemas de armas nuevas hasta el momento no han sido considerados específicamente por el derecho internacional humanitario. Sí han sido objeto de regulación por el derecho aeronáutico, pero no en cuanto al conflicto armado, materia de la que se ocupa el derecho internacional público.

Es importante resaltar que el uso de este tipo de armamento en Colombia no tiene antecedentes. Solo recientemente las Fuerzas Militares han empezado a utilizar pequeños ART, lanzados desde catapultas, con funciones de vigilancia e inteligencia (El Tiempo, mayo 2013). No obstan- 
te, la legalidad del uso de las ART en el conflicto armado colombiano todavía no está clara, por lo que es importante considerar todas las posibles variables, es decir, si están o no permitidas y si su uso es legal. Lo anterior con el propósito de eliminar el riesgo de conducir operaciones con este tipo de armas y trasgredir normas internacionales que regulan los conflictos armados. A la fecha no existe información sobre la utilización de drones armados en las operaciones en contra de grupos armados al margen de la ley. Para dar mayor profundidad se estudiará el uso de ART en Colombia y en Latinoamérica.

\section{AERONAVES REMOTAMENTE TRIPULADAS EN COLOMBIA Y LATINOAMÉRICA: ¿POR QUÉ Y PARA QUÉ?}

\section{A. Las ART en Latinoamérica}

Para comprender la necesidad de la utilización de ART en Colombia es importante estudiar cómo los drones han sido usados por otros países en la región. Bajo este entendido, es claro que Colombia no es el único país en el mundo interesado en la utilización de vehículos aéreos no tripulados por sus fuerzas militares. Brasil, Chile, Argentina, Ecuador, Venezuela y México han incursionado en la compra y uso de diferentes clases de drones (El Mercurio, 2013). Inclusive, muchos de estos países están desarrollando esta tecnología para uso propio (vigilancia y control de fronteras) y exportación (El Mercurio/GDA, 2012). El Hermes 900 fue adquirido por Chile para misiones de apoyo aéreo cercano y bom- bardeos (El Mercurio, 2013) y Colombia para la Fuerza Aérea (Webinfomil.com, 2012).

Si bien la mayoría de países de la región usan este tipo de aeronaves para funciones de vigilancia, existen estudios que demuestran que en diez años utilizarán drones armados (Freidersdorf, 2014) por los beneficios que ofrecen frente a las aeronaves convencionales, especialmente al eliminar el riesgo de perder un piloto entrenado en combate (Tucker, 2014). Es evidente, además, que el uso de ART reduce los costos económicos y humanos para las fuerzas militares de cualquier país, dado que incrementa la eficiencia y cobertura que puede tener una sola aeronave (Bowden, 2013).

\section{B. Las ART en Colombia}

No es secreto para nadie que el uso de ART en Colombia está direccionado mayoritariamente a atender las amenazas derivadas del conflicto armado interno. Tampoco es secreto que la Fuerza Aérea Colombiana (FAC) ha jugado un papel fundamental en el escalamiento del conflicto hacia los grupos armados al margen de la ley, y en su debilitamiento. Teniendo esto en cuenta, queda claro que el país puede asignar dos roles principales a las ART, dependiendo de las características de estas: (i) vigilancia, y (ii) apoyo aéreo cercano, bombardeo y destrucción de blancos selectivos, es decir una función bélica.

Como ya se señaló, las operaciones que pueden conducir las Fuerzas Armadas están limitadas a las características propias de cada drone. No existe mayor información pública acerca del tipo 
de ART que posee Colombia, lo que sí es sabido es que hasta el momento opera aeronaves Scan Eagle con propósitos de vigilancia (Webinfomil. com, 2013). Estas aeronaves son de fabricación estadounidense, su tamaño es relativamente pequeño, son lanzadas por catapultas y operadas desde tierra.

El gobierno colombiano ha mostrado interés en la adquisición de ART para operaciones de apoyo aéreo cercano, bombardeos y destrucción de blancos selectivos. Por ello, recientemente adquirió un número no especificado de Hermes 900 de fabricación israelí, con capacidad para portar y entregar armamento como misiles y bombas de diferentes clases (Webinfomil.com, 2012). De hecho, la fac está desarrollando paralelamente bombas inteligentes con una menor concentración de explosivos para que la onda expansiva sea significativamente menor, que pueden ser lanzadas desde las nuevas ART y dirigidas hacia sus objetivos en tierra (EI Tiempo, octubre 2013).

Lo anterior parecería indicar que las ART armadas van a ser utilizadas en Colombia de la misma manera que las aeronaves militares tradicionales, es decir, para operaciones en contra de grupos armados ilegales - por ejemplo, destrucción de campamentos-y apoyo aéreo cercano a tropas del Ejército, Policía e Infantería de Marina. Hasta el momento no se conoce que esto haya ocurrido, pero el hecho que la FAC adquiera este tipo de ART y desarrolle bombas inteligentes es indicio claro del uso que se les dará. Lo anterior se debe someter a las normas de los conflictos armados, por lo que, como señaló Bowden, el uso de drones armados para la lucha del crimen organizado resulta altamente ilegal (2013).

\section{Riesgo del uso de las ART en Colombia}

Con base en lo anterior, se identifica que el uso de drones en Colombia acarrea una serie de riesgos muy grandes. El primero de ellos es que su uso armado en el conflicto armado interno no se adecúe al derecho internacional humanitario y el Gobierno y sus fuerzas armadas se vean inmersas en violaciones al artículo 8 del Estatuto de Roma.

Otro riesgo que enfrenta el Gobierno colombiano es que mediante operaciones con ART armadas se incurra en muertes y lesiones a personas civiles, al igual que daños a propiedad civil, el medio ambiente y bienes culturales; que no sean necesarios para los propósitos de la misión o que no representen una ventaja militar significativa y sí lo impliquen en investigaciones y juicios por la comisión de crímenes de lesa humanidad y crímenes de guerra, contemplados en los artículos 7 y 8 , respectivamente, del Estatuto de Roma.

Adicionalmente, si los drones armados son utilizados en desarrollo de operaciones legales especialmente en contra del crimen organizado, Colombia podría incurrir en graves violaciones al derecho internacional, puesto que el uso de la fuerza es muy distinto en situaciones de conflicto armado que en contextos de lucha contra el crimen. Como tal, la utilización de drones armados para combatir grupos criminales implicaría la trasgresión del régimen internacional de 
derechos humanos, específicamente el artículo 6 del Pacto Internacional de Derechos Civiles y Políticos. Este dicta que el derecho a la vida es inherente al ser humano, y fue desarrollado por el Octavo Congreso de las Naciones Unidas sobre la Prevención del Delito y Tratamiento del Delincuente, de 1990, el cual en sus disposiciones especiales estatuyó:

Los funcionarios encargados de hacer cumplir la ley no emplearán armas de fuego contra las personas salvo en defensa propia o de otras personas, en caso de peligro inminente de muerte o lesiones graves, o con el propósito de evitar la comisión de un delito particularmente grave que entrañe una seria amenaza para la vida, o con el objeto de detener a una persona que represente ese peligro y oponga resistencia a su autoridad o para impedir su fuga, y sólo en caso de que resulten insuficientes medidas menos extremas para lograr dichos objetivos. En cualquier caso, solo se podrá hacer uso intencional de armas letales cuando sea estrictamente inevitable para proteger una vida. (Cursivas añadidas).

La trasgresión a las disposiciones mencionadas implicaría el sometimiento del Gobierno y sus fuerzas militares a la jurisdicción de tribunales internacionales como la Corte Interamericana de Derechos Humanos y la Corte Penal Internacional.

\section{EL DERECHO INTERNACIONAL HUMANITARIO Y LAS ART}

\section{A. Legalidad del uso de armas en los conflictos armados}

Antes de proceder al análisis sobre la legalidad del uso de armas, se debe aclarar que en este texto se hace referencia al armamento convencional, y no a las armas de destrucción masiva. Estas últimas reciben un tratamiento especial por parte del derecho internacional, que no es relevante al hacer un estudio sobre la legalidad del uso de ART en los conflictos armados.

La restricción al uso de cierto tipo de armamento y municiones en los conflictos armados viene desde la Convención de San Petersburgo de 1868, en cuyo preámbulo se introdujo por primera vez la prohibición de causar sufrimiento innecesario al enemigo, también conocida como la Cláusula Martens, transversal a la gran mayoría de instrumentos de derecho internacional humanitario. Esta reza:

En los casos no previstos en el presente Protocolo o en otros acuerdos internacionales, las personas civiles y los combatientes quedan bajo la protección y el imperio de los principios del derecho de gentes derivados de los usos establecidos, de los principios de humanidad y de los dictados de la conciencia pública (Dinstein, 2004).

Los avances acá dispuestos fueron retomados y desarrollados por las regulaciones de La Haya de 1899 y 1907. Adicionalmente, los Convenios 
de Ginebra de 1949 y sus Protocolos Adicionales profundizaron en la prohibición de cierto tipo de armas en las normas de los conflictos armados. Estos instrumentos prohibieron por completo el uso de veneno y de proyectiles explosivos y de fácil expansión para armamento de infantería, es decir, armas individuales. Adicionalmente, se introdujo la restricción de adelantar ciertos métodos para librar la guerra (Dinstein, 2004, pp. 63-64).

En 1980 se firmó el Convenio sobre ciertas Armas Convencionales, por medio del cual se limitaba expresamente el uso de ciertas clases de armamento cuya utilización no podía hacer distinción entre un combatiente y un no combatiente. Mediante este Convenio y sus protocolos se logró la prohibición o restricción de uso de: (i) armas de fragmentación cuyos elementos no pudiesen ser detectados por rayos $X$, (ii) minas antipersonales y trampas antipersonales, ${ }^{4}$ (iii) armas incendiarias, ${ }^{5}$ y (iv) armas láser que causen ceguera. Este Convenio fue complementado por el Tratado de Ottawa de 1997, por medio del cual se prohíbe por completo el uso de minas antipersonales. Adicionalmente, en la Convención de Dublín de 2008 se prohibió por completo el uso de municiones de racimo (Dinstein, 2004, pp. 64-73).

Se evidencia que las armas que están explícitamente prohibidas por el derecho internacional

4 Por trampa antipersonal se entiende aquel dispositivo no explosivo que se esconde para que la víctima caiga en ella sin antes poder preverlo.

5 Armas que utilizan mezclas de químicos como el fósforo para generar fuego, y causar daño y destrucción a través de este. humanitario son aquellas cuyo operador no puede dirigirlas hacia un objetivo específico, o que causan daños innecesarios más allá de cualquier ventaja militar. Es decir, que por su naturaleza trasgreden el principio de distinción del derecho internacional humanitario y atentan contra la prohibición de causar daños innecesarios. Se observa entonces que la razón para prohibir el uso de estos armamentos radica en su naturaleza extremadamente destructiva e indiscriminada, mas no en la manera como son usadas.

En consecuencia, para determinar si una clase de armamento es violatoria de las normas de los conflictos armados se debe atender a un estudio objetivo de sus características (tamaño, munición, tripulación) y no la manera como puede llegar a usarse (en operaciones de destrucción de blancos selectivos, sobre centros urbanos poblados, en contra de grupos armados al margen de la ley), pues esta parte, que constituye el estudio subjetivo, corresponde al derecho penal internacional. Específicamente el artículo 8(2) (b)(xx) del Estatuto de Roma establece lo que es considerado crimen de guerra:

Emplear armas, proyectiles, materiales y métodos de guerra que, por su propia naturaleza, causen daños superfluos o sufrimientos innecesarios o surtan efectos indiscriminados en violación del derecho internacional de los conflictos armados, a condición de que esas armas o esos proyectiles, materiales o métodos de guerra, sean objeto de una prohibición completa. (Cursivas añadidas).

Por lo explicado anteriormente, es claro que no existe prohibición en el derecho internacional 
humanitario respecto al uso de ART en el conflicto armado. Sin embargo, dado que se ha sostenido que este tipo de armamento debe ser considerado como una aeronave, es necesario revisar la normatividad que regula el combate aéreo. La norma más importante al respecto son las Reglas de La Haya sobre el Combate Aéreo, de 1922. El artículo XVII de este instrumento hace extensible al combate aéreo las normas de la Convención de Ginebra de 1906, y como tal la Cláusula Martens. Igualmente, el artículo XXII prohíbe el bombardeo de población civil con el propósito de aterrorizarla, y proscribe el uso de este tipo de armamento en contra de no combatientes y bienes civiles. Adicionalmente, el artículo XXIV dispone que el bombardeo aéreo solo sea legítimo cuando se direccione en contra de objetivos que generen ventaja militar para el atacante, y restringe el bombardeo de pueblos y centros urbanos a situaciones de concentración militar suficiente que justifique la acción. Por otra parte, el artículo XXV establece la obligación de limitar cualquier tipo de daño colateral a bienes civiles, culturales y médicos.

Es decir, las Reglas de La Haya sobre el Combate Aéreo hacen extensibles las normas del derecho internacional humanitario a la utilización de aeronaves en los conflictos armados. Igualmente, cabe señalar que de existir lagunas en la regulación del combate aéreo se debe aplicar una analogía con las regulaciones del combate terrestre, el combate naval y el derecho internacional humanitario (Ronzitti, 2006, p. 15). Teniendo en cuenta que se ha afirmado que las ART Son aeronaves, se puede sostener que las Reglas de La Haya las cobijan y, como tal, se les aplican los mismos principios del derecho internacional humanitario (DIH). Sin embargo, no se encuentra ningún indicio que señale que el uso de ART esté prohibido por las normas que regulan la legalidad del uso de armas en los conflictos armados.

Es importante recalcar que Colombia ha adherido a todos los instrumentos internacionales que hasta este punto se han mencionado.

\section{B. Principio de distinción}

Para empezar, es necesario determinar qué es el principio de distinción al que hace referencia el derecho internacional humanitario. Este señala que los combatientes tienen la obligación de distinguir entre objetivos militares y población civil protegida. Este principio está inmerso en los artículos 50 y 51 del capítulo II del Protocolo Adicional I de las Convenciones de Ginebra de 1949, el capítulo III de este mismo texto y el artículo 8 del Estatuto de Roma. Adicionalmente, el principio de distinción es considerado una norma de derecho internacional humanitario consuetudinario, por lo que la no adhesión a los Protocolos Adicionales o al Estatuto de Roma no excluye la obligación de un Estado de cumplir sus disposiciones (Comité Internacional de la Cruz Roja, 2014a).

Para ampliar sobre el principio de distinción, se señala que un no combatiente es cualquier persona que no desarrolla actividades militares en el contexto de un conflicto armado. En contraposición, un miembro de un grupo armado es una persona, militar o miembro de un grupo 
armado organizado. A pesar de esta diferencia, lograr una distinción clara ha resultado complicado en muchos contextos (Melzer, 2009). Para resolver esto, el Comité Internacional de la Cruz Roja (CICR) ha hecho una diferenciación con base en la participación directa en las hostilidades, según se trate de conflictos armados internacionales y conflictos armados internos. El artículo 3 común de las Convenciones de Ginebra de 1949 establece que las partes de un conflicto armado tienen la obligación de proteger a las personas que no participan en las actividades hostiles, lo que incluye a los combatientes que han depuesto las armas, y que como tal han adquirido el estatus de hors de combat. ${ }^{6}$ Se desprende de esto que la obligación de proteger a la población civil aplica tanto a actores estatales como a no estatales (Melzer, 2009, p. 28).

En cuanto a los conflictos armados internos, el Protocolo Adicional II dispone que las fuerzas armadas, las fuerzas armadas disidentes y otros grupos armados organizados tienen capacidad para desarrollar operaciones militares de manera sostenida y concertada. Por lo tanto, les son aplicables las obligaciones del derecho internacional humanitario (Melzer, 2009, p. 29).

De acuerdo con Melzer (2009), los actores estatales en un conflicto armado interno son las fuerzas armadas regulares (p. 31). Los miembros de las fuerzas armadas regulares y su pertenencia a estas se regulan por el derecho doméstico, al igual que la identificación de uniformes, insignias y equipamiento. Adicionalmen-

6 Puesto fuera del combate. te, esta categoría no diferencia entre naturaleza civil o militar, siempre y cuando desarrolle operaciones militares de manera sostenida (p. 31). Las fuerzas armadas disidentes, al igual que los grupos armados organizados, son actores no estatales dentro de un conflicto armado interno. Las primeras, las fuerzas armadas disidentes, son organismos con estructuras jerárquicas que anteriormente tuvieron un vínculo con fuerzas armadas regulares (p. 32). De la misma forma, los grupos armados organizados reclutan a sus miembros directamente de la población civil y su existencia no se ajusta al derecho nacional (p. 32). Para determinar la pertenencia de una persona a estos grupos, es necesario aplicar un criterio de continuidad en el desarrollo de la función militar, es decir, una función de combate permanente (p. 33).

Teniendo esto en cuenta, se puede afirmar que la manera más efectiva de identificar a un civil es determinando que no clasifica en ninguna de estas categorías. Las personas que no son parte de estos grupos o que depusieron sus armas gozan de la protección del derecho internacional humanitario. Por tanto, un civil puede participar directamente de las hostilidades y perder su protección (Melzer, 2009, p. 44).

Como ya se ha mencionado, el principio de distinción conlleva la obligación para los combatientes de diferenciar entre un objetivo militar y la población civil. Esta obligación está presente en la gran mayoría de instrumentos de derecho internacional humanitario, sin embargo cobra más importancia en los Convenios de Ginebra y el Protocolo Adicional II aplicable a los conflic- 
tos internos, por la misma naturaleza de este tipo de conflagraciones. En dichos conflictos los combates ocurren en espacios en donde puede habitar población civil que puede resultar afectada directamente.

El anterior es un riesgo presente en la gran mayoría de conflictos internos, entre ellos el colombiano. Surge entonces el interrogante sobre qué puede ocurrir si en el desarrollo de una operación militar muere población civil. Para responder se debe recalcar que el principio de distinción prohíbe atacar directamente a los civiles o a los bienes civiles. No obstante, hay ocasiones en que la muerte de civiles o la destrucción de un bien civil son inevitables aun cuando se practiquen las operaciones con la diligencia que demandan. Estas muertes y afectaciones a bienes civiles, llamados daños colaterales, son permitidos por el derecho internacional humanitario siempre y cuando se demuestre que no existía intención de causarlos, que eran necesarios para obtener una ventaja militar y que la operación se desarrolló con la debida diligencia (Solis, 2010, p. 253). Sin embargo, no es posible conducir operaciones militares directamente en contra de bienes civiles bajo la justificación de obtener una ventaja militar. Esto fue reiterado por el Tribunal Penal Internacional para la ex Yugoslavia en el caso Galić, donde estableció que “Bajo ninguna circunstancia la necesidad militar justifica ningún abuso de las prohibiciones generales sobre ataques a civiles y objetos civiles" (Fiscalía vs. Stanislav Galić, 2003).

Por consiguiente, antes de realizar una operación militar los comandantes y dirigentes tienen la obligación de estudiar cómo puede resultar afectada la población civil y poner en una balanza dicha afectación y la ventaja militar que pueden obtener. En caso de ser mayor la afectación a la población civil, el comandante debe desistir. Adicionalmente, si no es posible distinguir si se trata de un civil o un combatiente se debe asumir que se trata de un civil (Melzer, 2009).

La prohibición de atacar personas y bienes civiles se enmarca en el artículo 13(2) del Protocolo Adicional II y en el artículo 3(2) del Protocolo II Revisado sobre Ciertas Armas Convencionales, y ha sido incorporada en los manuales militares de la gran mayoría de países (Comité Internacional de la Cruz Roja, 2014a). Adicionalmente, el Tribunal Penal Internacional para la ex Yugoslavia estableció que para que se constituya una violación al principio de distinción, los actores del ataque deben conocer expresamente que se estaba atacando a no combatientes (Fiscalia vs. Tihomir Blaskić, 2000). Como tal, para que se produzca una violación al principio de distinción debe existir una intención clara de causar daños a la población civil.

Por otra parte, la Corte Internacional de Justicia ha establecido que se prohíbe el uso de armas que causen efectos indiscriminados. En vista de esto señaló que mediante la Cláusula Martens, mencionada anteriormente, se “[p]rohibió [...] algunos tipos de armas, bien por sus efectos indiscriminados para los combatientes y los civiles, bien por los daños innecesarios (sufrimientos innecesarios) causados a los combatientes, es decir, por ocasionar daños mayores que los inevitables para conseguir objetivos militares le- 
gítimos (Corte Internacional de Justicia, 1996). (Cursivas añadidas).

De esta manera, se reitera lo sostenido hasta el momento en cuanto a que se prohíbe el uso de cualquier tipo de arma que no pueda distinguir entre un objetivo civil y un objetivo militar.

Volviendo sobre las características técnicas de Ias ART, recordemos que estas pueden distinguir claramente sus objetivos. De hecho, gracias a la capacidad de vigilancia permanente (McNab y Matthews, 2011) y su alta autonomía de vuelo pueden permanecer circundando una cierta área por un largo periodo de tiempo, permitiendo que analistas estudien el objetivo y determinen si se debe proceder o no con la operación (Bowden, 2013). No obstante, hay quienes sostienen que la calidad de las cámaras utilizadas en este tipo de aeronaves no permite hacer distinciones apropiadas y, como tal, conllevan el riesgo de realizar ataques en contra de la población civil. Al respecto resulta útil el estudio realizado por la Stanford International Human Rights and Conflict Resolution Clinic (IHRCRC) y Global Justice Clinic (GJC), Facultad de Derecho de la Universidad de Nueva York, titulado Living Under Drones: Death, Injury, and Trauma to Civilians from US Drone Practices in Pakistan, publicado en inglés en septiembre de 2012.

En este se señala que los operadores de drones disparan sin tener certeza sobre la identidad de su objetivo; que los drones causan una serie de daños que no son contemplados por los gobiernos, entre ellos los daños psicológicos a la población civil ocasionados por su sobrevuelo constante sobre un área y los disparos en contra de no combatientes; que los daños psicológicos causados por los drones son indiscriminados, puesto que no distinguen entre personal combatiente y población civil; que en Pakistán los drones han sido utilizados en ataques de doble toque (double-tap attacks), esto es, que se hace un primer bombardeo sobre un objetivo, para luego volver a atacar cuando se acerca el personal de rescate. Con base en lo anterior, este reporte también sostiene que el uso de drones no respeta el derecho internacional humanitario.

No obstante, existen doctrinantes que sostienen que la utilización de drones es la forma más humana de desarrollar la guerra. Según Lewis (2013), desde que se empezó a usar drones se redujo el número de bajas civiles y como tal la dimensión de los daños colaterales, pues la capacidad de seguir y observar el blanco le permite al operario tomar la decisión de disparar en circunstancias que no pongan en riesgo a la población civil, y el hecho de que sean operados remotamente posibilita que abogados, analistas de inteligencia y comandantes del más alto rango analicen la situación antes de disparar, asegurando siempre un mayor nivel de distinción.

Otra crítica al uso de ART en el reporte Living under drones y por los académicos McNab y Matthews (2011), es que dado que el operario se encuentra abstraído de la zona de combate, pierde la noción de que está operando una aeronave con la capacidad de destruir vidas. Este efecto es conocido como playstation, en razón a que el piloto puede llegar a sentirse operando 
una consola de video juegos y pensar que sus acciones no tendrán consecuencias en el mundo real (Lewis, 2013). Según esta posición, el juicio del operario se ve perturbado por las características propias de la operación del drone.

Por otra parte, existe evidencia de que los pilotos de ART sufren mayor estrés de combate y postraumático debido a que como sobrevuelan y observan a su objetivo permanentemente, Ilegan a conocer sus hábitos de vida y a generar cierta familiaridad (Bowden, 2013). De hecho, los efectos psicológicos sufridos por los pilotos de drones son mucho mayores que los que padecen las tropas de infantería, situación que deja sin piso la crítica de que los pilotos pierden sentido de la realidad (Lewis, 2013). De acuerdo con esto, los pilotos de ART no solo tomarían más precauciones antes de disparar, sino que prestarían mayor atención al principio de distinción, hecho que desatiende el efecto playstation.

En conclusión, no es evidente que el uso de ART en sí mismo atente contra el principio de distinción. Inclusive, hay quienes defienden que sus operarios toman mayores precauciones, y como tal su utilización implica una mayor capacidad de discernir sobre el objetivo que la que se puede llegar a tener con una aeronave militar tradicional.

\section{Principio de proporcionalidad}

Este principio del derecho internacional humanitario hace referencia a la obligación que tienen los actores armados de un conflicto, de reducir la afectación a personas civiles a su máxima expresión. Es decir, debe existir una relación de proporcionalidad entre la ventaja militar obtenida por una operación y los daños causados a la población civil, en otras palabras, la ventaja militar obtenida tiene que ser siempre proporcionalmente más grande que los daños colaterales causados. Sin embargo, este principio ha sido generalmente malinterpretado por razones semánticas y por el uso que se le da en otras ramas del derecho. Así, Yoram Dinstein afirma:

La actual desproporción de la tasa de víctimas entre civiles y combatientes es completamente inaceptable. Cualquier persona que esté mínimamente interesada en el derecho internacional humanitario debe buscar reducir el número de víctimas civiles en la guerra. No obstante, este objetivo debe ser el de minimizar el número de víctimas civiles, no de erradicarlas como tal. No hay forma alguna de poder eliminar la muerte y lesiones a civiles, dado que existe el daño colateral, los errores, los accidentes y la pura mala suerte (2002, p. 219). ${ }^{7}$

Así las cosas, la primera característica del principio de proporcionalidad es que solo aplica a afectaciones a no combatientes. Por lo tanto, nunca habrá violación al principio de proporcionalidad cuando luego de un ataque solo resulten afectados objetivos militares. De ahí que el Protocolo Adicional I, aplicable a los conflictos internacionales, establezca que la proporcionalidad debe recaer sobre el nivel de daños causados a no combatientes, y sobre la ventaja militar obtenida por un ataque. Así, si los daños a

7 Traducción realizada por el autor de este texto. 
los no combatientes superan la ventaja militar obtenida, la operación resultará ilegal (Solis, 2010, p. 273).

Si bien este texto se ocupa de la utilización de cierto tipo de armas en conflictos armados internos, se debe señalar que el Protocolo Adicional II, aplicable a esta clase de conflictos, no regula expresamente el principio de proporcionalidad. No obstante, este resulta aplicable dado que el Comité Internacional de la Cruz Roja ha señalado que el principio de proporcionalidad enmarcado en el Protocolo Adicional I, es un principio de la humanidad derivado del derecho internacional humanitario consuetudinario. Esto también ha sido reiterado por tribunales internacionales como el Tribunal Penal Internacional para la ex Yugoslavia y la Comisión Interamericana de Derechos Humanos (Comité Internacional de la Cruz Roja, 2014b).

Los tribunales internacionales han sostenido que si bien el principio de proporcionalidad no está incluido en los instrumentos aplicables a los conflictos internos, es aplicable a ellos. El Tribunal Penal Internacional para la ex Yugoslavia se ha pronunciado en este sentido a lo largo de los fallos de los casos Galić, Martić y Kupreskić. En el sistema interamericano, la Comisión Interamericana de Derechos Humanos señaló en el Tercer reporte sobre la situación de derechos humanos en Colombia (1999), que la regla de proporcionalidad tiene un rol importante en los conflictos armados internos, y de esa manera aplica a todos los actores armados del conflicto. Adicionalmente, señala que los daños colaterales no se pueden constituir en una justificación para la destrucción arbitraria de bienes civiles.

Dado que el principio de proporcionalidad implica una obligación para los combatientes, sus operaciones deben ser lo más certeras posibles para minimizar daños colaterales. Esto no significa que los combatientes deben responder de manera proporcionada al ataque de su enemigo (Solis, 2010). Esta concepción de la proporcionalidad hace parte del derecho internacional público y el sistema internacional de derechos humanos, y dista de la concepción ofrecida por el derecho internacional público. De acuerdo con Hoge, para determinar si un ataque es proporcional o no, no basta con identificar el número de víctimas civiles que hubo, sino que se debe balancear contra la ventaja militar obtenida. Esto implica que no existe una regla para determinar cuándo un ataque es proporcional o no, en consecuencia, el estudio debe realizarse caso por caso (citado por Solis, 2010, p. 277).

Esto quiere decir que el principio de proporcionalidad es aplicable a las formas y métodos de hacer la guerra, más allá que al armamento utilizado. No obstante, existen armas que facilitan la conducción de operaciones más proporcionadas que otras, dado que por su naturaleza son más precisas e implican daños colaterales significativamente menores (Lewis, 2013).

Teniendo en cuenta las características técnicas de las ART, es posible afirmar que estas aeronaves están diseñadas para asegurar la mayor precisión en las operaciones que desarrollan. Gracias a la capacidad de vigilancia permanen- 
te, las cámaras y sistemas de video que utilizan y las bombas dirigidas por láser, pueden reducir significativamente las afectaciones a la población civil por un bombardeo o una operación de destrucción de un blanco selectivo. Consecuentemente, este tipo de aeronaves pueden ser utilizadas para reducir el nivel de daños colaterales (Bowden, 2013).

Sin embargo, el reporte Living under drones muestra cómo los ataques de doble toque hacen que los bombardeos con drones violenten el principio de proporcionalidad (Stanford Law School y NYu School of Law, 2013). En este tipo de ataques, las primeras víctimas son usadas como "carnada" para atraer el personal de rescate y maximizar los daños a los objetivos. No obstante, el problema en este caso no es el tipo de armamento usado sino la estrategia. Con esto en mente, es perfectamente posible causar el mismo daño con un ataque de doble toque utilizando una ART o una aeronave tradicional.

Lo que sí es posible es que al tratarse de una operación remota de la aeronave se llegue a deshumanizar a los objetivos y a disminuir la precaución de velar por la protección de la vida de personas civiles, lo cual daría sentido al efecto playstation tan fuertemente criticado por autores como Bowden y Lewis. Estudios han demostrado que los pilotos de ART, efectivamente, sufren un mayor estrés de combate, derivado de la presión que causa observar la vida de personas sobre una misma área. Aun así, no se encuentra que este argumento presente un riesgo significativo, si se toman las medidas para mitigarlo que se mencionan más adelante. De hecho, no existen suficientes pruebas de que efectivamente estas plataformas de armas deshumanicen la guerra y signifiquen un mayor riesgo de violación del principio de proporcionalidad.

En conclusión, el problema de la proporcionalidad radica en la forma de conducir la operación. Si bien el armamento usado puede implicar una reducción en los daños colaterales por razones de precisión, dicha reducción puede ser proporcional a lo que su operador decida. De esta suerte, los drones per se no resultan violatorios del principio de proporcionalidad.

\section{IV. ¿CUÁLES SON LOS RIESGOS DE OPERAR AERONAVES REMOTAMENTE TRIPULADAS?}

El reporte Living under drones contiene una fuerte crítica al uso de estas aeronaves para cumplir objetivos militares. A esta se suman otros autores que también han sostenido que los riesgos de usar ART armadas en los conflictos son mayores a los beneficios, como ya se señaló en este texto.

Aquí se hace referencia a los riesgos que implica la utilización de este tipo de armamento en contra de personas protegidas por el derecho internacional humanitario. No obstante, se trata del mismo riesgo en el que se incurre al utilizar cualquier tipo de armamento, pues en ambos corresponde al operario tomar las precauciones para que esto no suceda. Es decir, independiente del armamento, está prohibido dirigir intencionalmente ataques en contra de la población 
civil, así como los ataques de doble toque ocurridos en Pakistán (IHRCRC y GJC , 2012).

Otro riesgo en el que se incurre, según el reporte Living under drones, es el de generar daños psicológicos a la población civil que habita en las zonas donde operan las ART. Para evitarlo es necesario que el uso de drones se haga responsablemente, esto es, solo para responder a una amenaza clara, y los operarios no solo deben contar con la autorización de un superior responsable para poder entregar el armamento, sino que la decisión de disparar en contra de un objetivo debe ser consultada y verificada, con el propósito de que no se afecte la vida de personas civiles. Si se asegura la reducción de muertes civiles, la población civil no sufrirá el miedo que se tiene a este tipo de aeronaves en Pakistán. De nuevo, se trata de un riesgo derivado del uso, más que del armamento en sí.

Otro riesgo que ha sido identificado es la desensibilización de los operarios de las ART, producida, según algunos estudios como los mencionados anteriormente, por el hecho de que estos son abstraídos de la realidad y no sienten en carne propia lo que está ocurriendo en el campo de combate. Frente a esto, las fuerzas militares deben hacer un acompañamiento psicológico permanente a los operarios; adicionalmente, el Código Penal Militar y el Código Penal deben ser fortalecidos para agravar las penas por homicidios, lesiones y daños en bien ajeno causados por el uso irresponsable y negligente de drones, con el fin de servir de efecto disuasorio para este tipo de comportamientos; de igual forma, el requerimiento de tener que consultar cualquier ataque con un superior evita que el operario tome decisiones autónomas y arbitrarias.

Al respecto se debe señalar que si bien los anteriores riesgos presentan una amenaza a la violación del DIH, su naturaleza es más psicológica, es decir, tienen en cuenta la psiquis tanto de la población civil como de los operarios, lo cual excede el enfoque jurídico de este texto. Sin embargo, era necesario tenerlos en cuenta para poder ofrecer un estudio holístico de la situación y los riesgos que comporta la utilización de ART en conflictos armados.

No obstante lo anterior, existe evidencia de que los operarios de ART no sufren el llamado "efecto Playstation", sino que, por el contrario, sufren de estrés postraumático superior a soldados de tierra (Lewis, 2013). Aun así, las medidas anteriormente descritas pueden servir para evitar que se produzca este efecto bajo cualquier circunstancia.

Así, los riesgos aquí contemplados no se consideran altos en materia de peligro para la población civil si se toman las medidas necesarias y se emplean las ART de una manera que responda a los principios del derecho internacional humanitario. 


\section{V. ¿EL USO DE ART ARMADAS EN EL CONFLICTO ARMADO COLOMBIANO ES LEGAL Y CONSISTENTE CON EL DERECHO INTERNACIONAL HUMANITARIO?}

Con base en lo estudiado, se tiene que el uso de drones: (i) no está prohibido por las normas que regulan la legalidad de uso de ciertos tipos de armas en los conflictos armados; (ii) no está prohibido en las regulaciones sobre el combate aéreo; (iii) no atenta contra el principio de distinción, dado que sus características técnicas permiten que el operario tenga mayor certeza sobre su objetivo, y las armas que operan reducen los daños colaterales; (iv) tampoco atenta contra el principio de proporcionalidad, siempre y cuando el operario tome las precauciones debidas y siga la normatividad vigente.

En este sentido, el uso de ART armadas en el conflicto armado colombiano es legal y consistente con el derecho internacional humanitario, siempre que se respeten los presupuestos de uso de cualquier tipo de arma convencional y los medios de hacer la guerra, y no para la lucha contra el crimen. Así, se reafirma la posición de que el uso de drones es tan legal como cualquier tipo de arma, y como tal no debe estar sujeto a un régimen especial de derecho internacional (Carafano, 2013). Tampoco hay evidencia para señalar que el uso de drones armados en un conflicto resulte antiético (Anderson, 2013), aunque de nuevo esto excede el enfoque jurídico de este texto.

\section{CONCLUSIONES}

Las aeronaves remotamente tripuladas no se diferencian significativamente de las aeronaves militares tradicionales, por lo cual no requieren de regulación especial.

Las aeronaves remotamente tripuladas en Colombia tienen una alta probabilidad de ser usadas para operaciones de vigilancia, de apoyo aéreo cercano, de bombardeo y de destrucción de blancos selectivos, consistentes con los roles que ha tenido la Fuerza Aérea Colombiana en sede del conflicto armado vigente.

Los riesgos identificados no son significativamente altos, por lo cual se pueden tomar las medidas necesarias para prevenirlos y evitar que se viole la legislación doméstica y el derecho internacional humanitario.

La violación del derecho internacional humanitario se puede producir por la utilización de cualquier tipo de armamento convencional, si es utilizado en contra de los presupuestos de las normas de los conflictos armados y los medios para hacer la guerra. Es decir, el riesgo de trasgresión del derecho internacional humanitario es igual con la utilización indebida e ilegal de otros tipos de armamentos que con aeronaves remotamente tripuladas.

El uso de aeronaves remotamente tripuladas en roles bélicos, en sede de operaciones de lucha contra el crimen y aplicación de la ley, resulta violatorio del derecho internacional de los derechos humanos, y como tal constituye 
una grave contingencia para la fuerza pública colombiana.

El uso de aeronaves remotamente tripuladas en operaciones bélicas en sede del conflicto armado colombiano no es contrario al derecho internacional humanitario, y como tal pueden ser utilizadas por las fuerzas armadas.

\section{Referencias}

Anderson, K. (Mayo de 2013). Drones are ethical and effective. Oxford: Oxford University, Oxford Union.

Bowden, M. (14 de agosto de 2013). The Killing Machines. Obtenido de The Atlantic: http://www.theatlantic.com/magazine/archive/2013/09/the-killing-machines-howto-think-about-drones/309434/

Carafano, J. J. (26 de agosto de 2013). Say what you want about Drones - They're Perfectly Legal. Recuperado el 1 de mayo de 2014, de The Atlantic: http://www.theatlantic.com/ international/archive/2013/08/say-whatyou-want-about-drones-theyre-perfectly-legal/278740/

Castranova, M. C. (30 de junio de 2013). On Topic: The economics of drones. Recuperado el 24 de abril de 2014, de The Gazette: http:// thegazette.com/2013/06/30/on-topic-theeconomics-of-drones/

Cole, C. y Wright, J. (Enero de 2010). What are drones? Recuperado el 4 de marzo de 2014, de DronewarsUK.com: http://dronewars. net/aboutdrone/

Comisión Interamericana de Derechos Humanos. (1999). Tercer reporte sobre la situación de los derechos humanos en Colombia. Washington: $\mathrm{CIDH}$.

Comité Internacional de la Cruz Roja. (2011). El derecho internacional humanitario y el desafío de los conflictos armados contemporáneos. Ginebra: el Comité.

Comité Internacional de la Cruz Roja. (2014a). Rule 1. The Principle of Distinction between Civilians and Combatants. Recuperado el 1 de abril de 2014, de Customary IHL: http:// www.icrc.org/customary-ihl/eng/docs/v1_ cha_chapter1_rule1\#Fn_63_19

Comité Internacional de la Cruz Roja. (2014b). Rule 14. Proportionality in Attack. Recuperado el 1 de abril de 2014, de Customary IHL: http://www.icrc.org/customary-ihl/eng/ docs/v1_cha_chapter4_rule14

Corte Internacional de Justicia. (1996). Legalidad de la amenaza o el empleo de armas nucleares. La Haya: cı.

Dinstein, Y. (2002). Discussion: Reasonable Military Commanders and Reasonable Civilians. En A. Wall, Legal and ethical Lessons of NATO's Kosovo Campaign. Newport: Naval War College. 
Dinstein, Y. (2004). The Conduct of Hostilities under the Law of International Armed Conflict. Cambridge: Cambridge University Press.

El Mercurio/GDA. (20 de mayo de 2012). Drones se masifican en cielos de América Latina. Recuperado el 1 de mayo de 2014, de El País - Uruguay: http://www3.elpais.com. uy/120520/pinter-642091/internacional/ Drones-se-masifican-en-cielos-de-AmericaLatina/

El Mercurio. (5 de agosto de 2013). Los drones se masifican por el mundo y ya se debate reemplazar pilotos de combate.

El Tiempo. (10 de mayo de 2013). Más de 50 drones ya vuelan en Colombia. Recuperado el 29 de marzo de 2014, de El Tiempo: http://www.eltiempo.com/archivo/documento/CMS-12789712

El Tiempo. (12 de octubre de 2013). En menos de un mes, Fuerza Aérea estrena su 'drone' en operaciones. Recuperado el 12 de abril de 2014, de El Tiempo: http://www.eltiempo. com/archivo/documento/CMS-13117741

Freidersdorf, C. (7 de mayo de 2014). Lethal Drones: Coming Soon to Every Country That Wants Them. Recuperado el 8 de mayo de 2014, de The Atlantic: http://www.theatlantic.com/politics/archive/2014/05/lethaldrones-coming-soon-to-every-country-thatwants-them/361838/

Lewis, M. W. (21 de agosto de 2013). Drones: Actually the Most Humane Form of Warfare
Ever. Recuperado el 24 de abril de 2014, de The Atlantic: http://www.theatlantic.com/ international/archive/2013/08/dronesactually-the-most-humane-form-of-warfareever/278746/

McNab, M. y Matthews, M. (2011). Clarifying the law relating to unmanned drones and the use of force: the relationships between human rights, self-defense, armed conflict, and international humanitarian law. Denver Journal of International Law and Policy, XXXIX(4), 35.

Melzer, N. (2009). Interpretative guidance on the notion of direct participation in hostilities under international humanitarian law. Ginebra: International Committee of the Red Cross.

Ronzitti, N. (2006). The Codification of Law of Air Warfare. En M. Benkö, Essential Air and Space Law (p. 3-15). Ütrecht: Eleven International Publishing.

Solis, G. (2010). The Law of Armed Conflict. International Humanitarian Law in War. New York: Cambirdge University Press.

Stanford International Human Rights and Conflict Resolution Clinic (IHRCRC) y Global Justice Clinic (GJc), Facultad de Derecho de la Universidad de Nueva York. (Septiembre de 2012). Living Under Drones: Death, Injury, and Trauma to Civilians from US Drone Practices in Pakistan. 
Tribunal Penal Internacional para la ex Yugoslavia. (3 de marzo de 2000 y 5 de diciembre de 2003). Fiscalía vs. Stanislav Galiæ, IT-9829-T.

Tribunal Penal Internacional para la ex Yugoslavia. (2000). Fiscalía vs. Tihomir Blaskiæ, IT95-14-T.

Tribunal Penal Internacional para la ex Yugoslavia. (2003). Fiscalía vs. Stanislav Galić.

Tucker, P. (6 de mayo de 2014). Every Country Will Have Armed Drones Within Ten Years. Recuperado el 8 de mayo de 2014, de Defense One: http://www.defenseone.com/technology/2014/05/every-country-will-have-armeddrones-within-ten-years $/ 83878 /$ ?oref $=d$ river

Vogel, R. (2011). Drone Warfare and the Law of Armed Conflict. Denver Journal of International Law and Policy, XXXIX(1), 101-138.
Webinfomil.com. (6 de agosto de 2012). Colombia adquiere aeronaves no tripuladas UAV Hermes a Israel. Recuperado el 22 de abril de 2014, de Webinfomil: http://www.webinfomil.com/2012/08/colombia-adquiere-aeronaves-no.html

Webinfomil.com. (8 de marzo de 2013). Nuevas Aeronaves No Tripuladas NightEagle y ScanEagle para la Fuerza Aérea Colombiana. Recuperado el 6 de marzo de 2014, de Webinfomil: http://www.webinfomil.com/2013/03/ nuevas-aeronaves-no-tripuladas.html

Webinfomil.com. (23 de julio de 2013). Fuerza Aérea Colombiana anuncia la llegada de nuevas aeronaves no tripuladas. Recuperado el 22 de abril de 2014, de Webinfomil: http://www.webinfomil.com/2013/07/fuerza-aerea-colombiana-anuncia-la.html 